\title{
Mass-spectrometric investigations of gas evolution from silicon in electron beam melting
}

\author{
Yu.A. Asnis ${ }^{1}$, P.I. Baranskii ${ }^{2}$, V.M. Babich' ${ }^{2}$, S.P. Zabolotin ${ }^{1}$, \\ Yu.G. Ptushinskii ${ }^{3}$, V.G. Sukretnyi ${ }^{3}$ \\ ${ }^{1}$ E.O. Paton Electric Welding Institute, NAS of Ukraine, \\ ${ }^{2} V$. Lashkaryov Institute of Semiconductor Physics, NAS of Ukraine, \\ ${ }^{3}$ Institute of Physics, NAS of Ukraine
}

\begin{abstract}
Method of mass-spectrometry with time-of-flight recording of the desorbed products was used to study the gas evolution of impurities from the subsurface layer of $\mathrm{Si}$ crystals molten by the electron beam (of $\sim 2 \mathrm{~mm}^{2}$ area) in the vacuum of $10^{-5}-10^{-7} \mathrm{~Pa}$. It is shown that irrespective of vacuum level, oxygen $(m=32)$ and hydrogen $(m=2)$ in the molecular state as well as $\mathrm{Si}$ atoms $(m=28)$ are registered as the main components of gas evolution in the mass-spectrum in melting. With longer time of the subsurface layer exposure in the molten state, an indication of CO evolution (fragment peak $m=12$ ) appears in the mass-spectrum. There is, however, a ground to believe that this is the consequence of gas evolution from the fixtures, and not from the Si sample. Features of gas evolution were revealed at the initial stage of heating and melting of Si sample, depending on the previous heat-treatment of the sample. If melting the subsurface zone proceeds after contact with the atmosphere, initial peaks of evolution of oxygen and hydrogen molecules and Si atoms are observed. These are partially weakened with further keeping the sample in the molten state. In our opinion, such a peak is due to contamination of the surface at such a contact. A longterm exposure in vacuum of a sample cooled after melting does not lead to appearance of the above peak at subsequent melting.
\end{abstract}

Keywords: silicon, gas evolution.

Manuscript received 11.01.06; accepted for publication 29.03.06.

\section{Introduction}

The important role of ultrahigh vacuum for cleaning silicon crystals from gas impurities in crucibleless recrystallisation of $\mathrm{Si}$ using the electron beam and allowing for the backflows from the environment is substantiated in [1]. Gas evolution from the crystal may have an essential influence on the composition and density of the gas atmosphere around the molten zone. Therefore, investigation of gas evolution from silicon in electron beam melting under different vacuum conditions is of considerable interest. In this case, it is important to know the composition of the desorption products, so that the mass-spectrometry procedure should be used. In this work, gas evolution from silicon was studied in the melt of a small part of the Si crystal surface treated with a focused electron beam.

\section{Experimental procedure}

Experimental procedure is schematized in Fig. 1. Unit design, means of achieving an ultrahigh vacuum in it, as well as the required measuring instruments in it are described in [2-4]. One of the essential features of this unit is simulation of the conditions of space infinity. Particles landing on the internal walls of the chamber, cooled by liquid nitrogen, and covered by a deposit of a fresh titanium film, are bound by them and do not distort the investigation results. This allows application of the procedure of single-flight mass-spectrometry for recording of the products of silicon gas evolution [2-4].

A cylindrical sample of $n$-Si:Sb single-crystal $\left(n_{e}=\right.$ $=10^{18} \mathrm{~cm}^{-3}, N_{0}=7 \times 10^{17} \mathrm{~cm}^{-3}$ ) of $8 \mathrm{~mm}$ diameter and $14.7 \mathrm{~mm}$ length was fastened in a standard electron beam evaporator, designed for evaporation of various metals and semiconductors in vacuum [5]. The principle of the action of such an evaporator (see Fig. 1) consists in heating of a rod of evaporation material (2) by electron beam (3). Electrons flying out of the gun (4) are turned through approximately $180^{\circ}$ by a lateral magnetic field of magnetic system (5) and are focused. Due to a good focusing of the electron beam, it heats (up to Si melting temperature: $T_{m}=1412 \pm 2{ }^{\circ} \mathrm{C}$ ) a subsurface zone of the sample of a small area and depth. Water cooling the copper crucible ensures heat removal and minimizes heat radiation of the structure. All of these 
features allow raising $\mathrm{Si}$ temperature up to $T_{m}$ in the point of electron beam focusing at negligible gas evolution from the surrounding fixtures. The molten zone may shift over the sample surface due to a change in the voltage applied between the sample and EB gun cathode. Mechanical feeding the evaporation material as the substance evaporates is also provided. Cooling of the chamber inner walls by liquid nitrogen and spraying of a fresh titanium film provided equivalent pumping speed of $\sim 1000 \mathrm{l} / \mathrm{s}$, and the level of vacuum in the chamber of $\sim 10^{7} \mathrm{~Pa}$ at substance melting, as well as neutralizing the adverse influence of the warm external walls of the chamber. In order to record the products of gas evolution from silicon at its local electron beam melting, time-offlight mass-spectrometer MCX-3A was used, in which the sensor is located in the region of direct flight of the particles at evaporation. Relative position of the evaporator with $\mathrm{Si}$ rod and MCX-3A ion source is shown in Fig. 1.

The automated system of recording the massspectra includes CAMAC crate (ADC F4226, BP305, crate-controller KK), master oscillator G5-56, time-offlight mass-spectrometer MCX-3A and PC. Respective software allows recording a range of mass-spectra, depending on one of the parameters (in this case, time of Si sample heating and melting).

Further processing the obtained data was performed using Origin 5.0 programs, this yielding both panorama three-dimensional dependencies in the co-ordinates of signal intensity $I$, time $t$ and mass number $m$, and other two-dimensional time dependencies of the selected mass peak intensity.

\section{Results and discussion}

Panoramic Fig. 2 shows how the spectrum as a whole changes at short-time local heating of a sample up to $T_{m}$. Maximum peak intensity is reached at the moment of melting of the sample subsurface zone (in a small region of the electron beam meeting with the Si crystal surface).

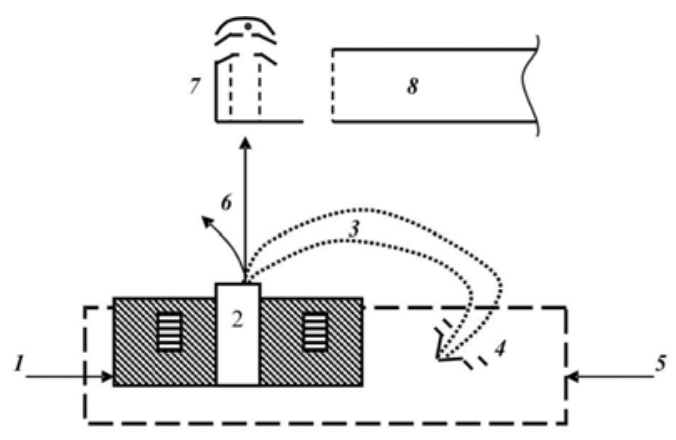

Fig. 1. Schematic of a relative position of the sample and the mass-spectrometer ion source. Numbers indicate: 1 - cooled crucible of the electron beam evaporator; 2 - silicon single crystal evaporated by the electron beam, 3 - electron beam, 4 - electron gun of the evaporator, 5 - magnetic system, 6 - evaporated particles, 7 - ion source of the time-of-flight mass-spectrometer, 8 - drift pipe.

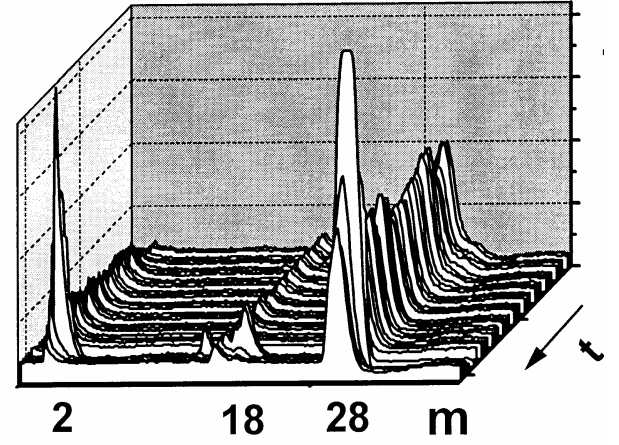

Fig. 2. Mass-spectrum panorama.

In the principal experiments, sample heating from room temperature up to $T_{m}$ occurs in approximately $10 \mathrm{~s}$, and then the temperature of the molten subsurface zone of $\sim 2 \mathrm{~mm}^{2}$ area remains constant $\left(T_{m}\right)$. The time for recording the maximum data file of 64 successive massspectra was $1.5 \mathrm{~min}$. Melt area was not completely stable, due to instability of emission current of electron beam evaporator. Software averaging of 10 neighbouring spectra was used to avoid the instability of spectra reproduction at unchanged conditions.

At an average vacuum level $\left(10^{-6} \mathrm{~Pa}\right)$, the residual gas spectrum (in the operation chamber) usually includes $\mathrm{H}_{2}(m=2)$, $\mathrm{CO}(m=28)$ and $\mathrm{H}_{2} \mathrm{O}(m=18)$ peaks. Obtained mass-spectra can be analyzed, knowing the percent ratio of the accompanying peaks from reference books (for instance, [6]). In our case, we compared $m=28$ with $\mathrm{CO}$, based on the presence of $m=12$ and $m=16$ fragments, and not with $\mathrm{N}_{2}(m=28)$, as $m=14$ fragment peak was absent from the residual gas spectrum obtained by us. Fig. 3 gives the time dependence of the amplitudes of the main peaks for a cold Si sample. Note that in the time interval required for recording the spectra files during the experiment, peak amplitudes of residual gases changed only slightly.

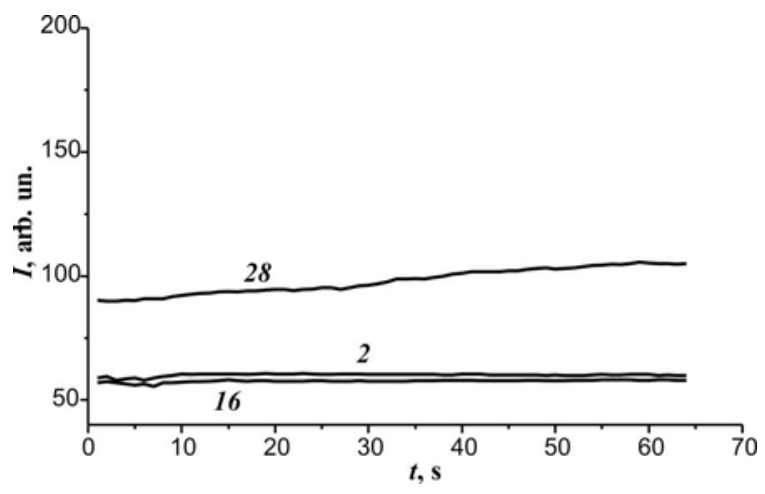

Fig. 3. Time dependence of mass-spectrum components in a cold sample. Mass numbers are given near each curve. 
Fig. 4 shows changes in peak amplitudes $(m=32$, 28, 16 and 2) at sample heating from the room temperature up to the melting one (first $10 \mathrm{~s}$ ) and subsequent maintenance of the sample zone in the melting mode $(10-90 \mathrm{~s})$. At the stage of sample heating from room temperature to $T_{m}$ (i.e. during $0-10 \mathrm{~s}$ interval), the intensity of $m=2,28,32$ peaks rises. Proceeding from the fact that in the operation chamber residual gases $\mathrm{O}_{2}(m=32)$ is absent, we may conclude that oxygen evolves from $\mathrm{Si}$ sample at the stage of sample heating up to melting. And the fact that the amplitude of $m=16$ peak remains unchanged in the range of experimental errors at an essential drop of molecular oxygen peak, suggests that it is not a fragment peak resulting from division of molecular oxygen. Moreover, by absolute value $m=16$ amplitude in Fig. 4 agrees with the data of Fig. 3.

Comparison of the data in Figs 3 and 4 may lead to another important conclusion: if $m=2\left(\mathrm{H}_{2}\right)$ and $m=16$ (O) in the residual gases of the working volume (at a cold Si crystal) are quantitatively represented almost in the same way (Fig. 3), at melting of Si crystal and practically unchanged $m=16$ value, $m=2\left(\mathrm{H}_{2}\right)$ value rather abruptly rises at the crystal melting stage (0 $10 \mathrm{~s}$ ) and begins to decrease only after $10 \mathrm{~s}$ (Fig. 4). Therefore, at Si melting not only molecular oxygen, but also molecular hydrogen evolve from the crystal.

Peak $m=28$, in principle, may be associated both with $\mathrm{CO}$ and with Si. However, $m=12$ fragment peak, which should accompany $\mathrm{CO}$, is absent in the massspectrum. Therefore, we believe that increase of $m=28$ peak in Fig. 4 (compared to Fig. 3) is due to $\mathrm{Si}$ evaporation. Data in Fig. 5 are another proof of that. This figure gives the spectra registered by a quadrupole mass-spectrometer, the sensor of which is located in the operation chamber beyond the line-of-sight zone of the Si crystal. It turned out that the amplitude of $m=28$ peak did not increase at crystal melting, which might not be in place, if $\mathrm{CO}$ made a significant contribution to the amplitude of $m=28$ peak in direct flight. A barely noticeable $m=12$ fragment peak appears only after 6 minute exposure of the molten zone (Fig. 5c), which may be associated with gassing of the heated fixture.

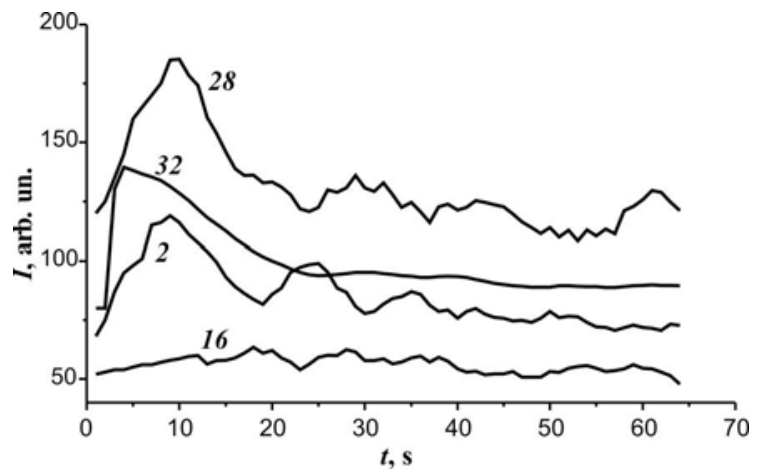

Fig. 4. Gas evolution in melting the Si sample. Mass numbers are given near the curves.
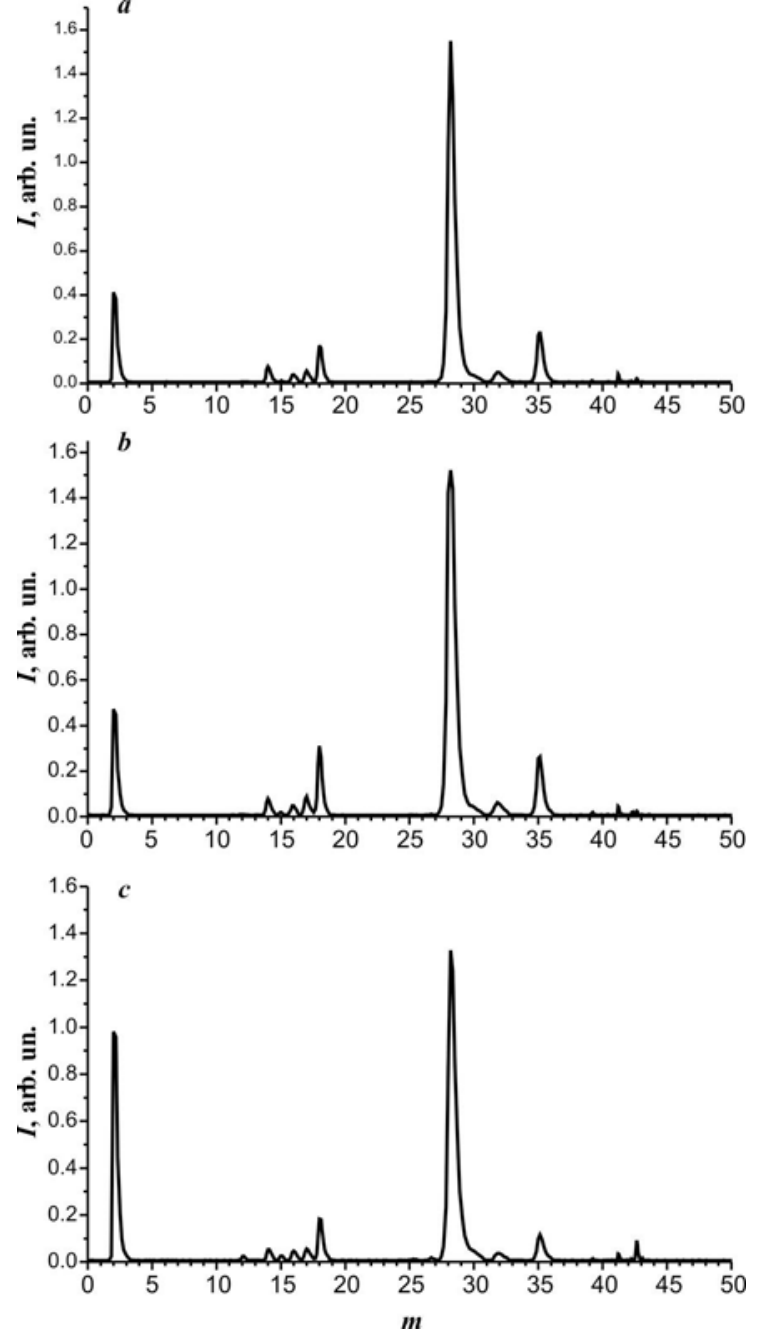

Fig. 5. Mass-spectra measured by the mass-spectrometer, the ion source of which is located beyond the line-of-sight zone relatively to the Si sample: a) cold Si crystal; b) exposure of the subsurface zone in the molten state for $2 \mathrm{~min}$; c) exposure in the molten state for 6 min.

As it seems from Fig. 5, the hydrogen peak $m=2$ markedly increased at crystal melting. This increase, most probably, results from hydrogen evolution from the Si crystal, although increase of background hydrogen level in the chamber due to fixture heating cannot be ruled out. At the initial stage of sample heating, when the fixture is still cold, increase of $m=2$ peak (Fig. 4) is certainly due to hydrogen evolution from the Si crystal. At further heating, the behaviour of the peak $(m=2)$ cannot be unambiguously related to gas evolution from Si bulk.

At sequential increase of the time of subsurface zone melting to $6 \mathrm{~min}$, a slight decrease is observed in the amplitudes of $m=2$, 32, 28 peaks, shown in Fig. 4 . At the total time of a small part of Si surface staying in the molten state, close to 2 hours, no other components of gas evolution were observed, except for the abovementioned, while the intensity of $m=32,16,2$ peaks decreased during this time by approximately an order of magnitude relatively to the initial stage. 
It was further observed that with increase of the time of the sample staying in the molten state initial increase of the peaks at melting after cooling, as shown in Fig. 4, is not observed. Such an increase is not renewed even after a rather long-time exposure of the cooled sample in high vacuum. However, the initial increase of oxygen and hydrogen peaks is found after the sample staying in air (chamber opening) or after a considerable increase of residual gas pressure (pumping interruption). Magnitudes of $m=2$, 32 peaks at sample melting remain almost constant or are slightly decreasing, and their intensities depend only on the total time of Si staying in the molten state. Thus, enhanced evolution of hydrogen and oxygen at the initial stage of Si sample melting is caused by some contamination of its surface at contact with the atmosphere.

Finally, let us consider the influence of vacuum conditions, under which Si melting proceeds, on the nature of gas evolution. An essential influence of the prior thermal conditions, under which the Si sample was kept, on the effectiveness of gas evolution at its subsequent melting, makes it more difficult to compare the data obtained under different conditions of vacuum. However, some conclusions seem obvious. First, the qualitative composition of the products that evolve at Si melting in vacuum of $\sim 10^{-7} \mathrm{~Pa}$ and $10^{-5} \mathrm{~Pa}$ does not differ (Fig. 6). We, unfortunately, cannot analyze the relationships of amplitudes of the respective peaks for different vacuum conditions as the relationship of arbitrary units in Figs 6a and $6 \mathrm{~b}$ is not known. However, it is possible and rational to compare the amplitudes within one figure. In Fig. $6 \mathrm{~b}$ $\left(10^{-7} \mathrm{~Pa}\right)$, amplitudes of $m=2$ and $m=32$ peaks (hydrogen and oxygen, respectively) are close to each other, while in Fig. $6 a\left(10^{-5} \mathrm{~Pa}\right) m=2$ amplitude is much
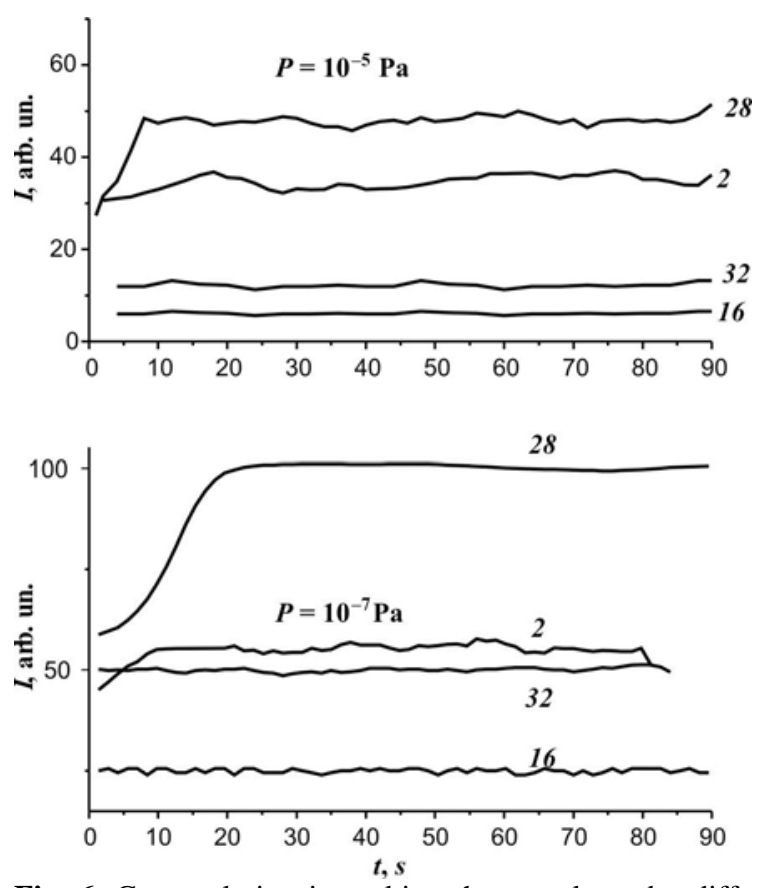

Fig. 6. Gas evolution in melting the sample under different vacuum conditions. higher than that of $m=32$. These facts indicate that hydrogen availability in the mass-spectrum is determined not only by its evolution from Si crystal, but also its presence in the residual gas composition.

\section{Conclusions}

1. The main components of gas evolution in melting of a subsurface Si layer by the electron beam under vacuum are molecular oxygen $m=32$ and hydrogen $m=2$. An increase of the amplitude of $m=28$ peak is also observed, but due to silicon evaporation, rather than to CO evolution from the molten sample. The latter conclusion is confirmed by experiments, in which the ion source of the second mass-spectrometer was located beyond the line-ofsight zone relatively to Si sample.

2. Qualitative composition of gas evolution does not depend on vacuum level in the range of $10^{-7}$ to $10^{-5} \mathrm{~Pa}$. However, the ratio of $\mathrm{O}_{2}$ and $\mathrm{H}_{2}$ components changes in favour of the latter at vacuum level lowering. This feature is indicative of the fact that the hydrogen source is not only hydrogen evolution from the molten subsurface layer of Si sample, but also gas evolution from the fixture at heating.

3. After several hours of exposure of the sample subsurface zone in the molten state, two such features of gas evolution were observed: 1) intensity of $\mathrm{O}_{2}$ and $\mathrm{H}_{2}$ evolution decreases by an order of magnitude and 2) after sample cooling in the high vacuum the initial growth of $m=32\left(\mathrm{O}_{2}\right)$ and $m=2\left(\mathrm{H}_{2}\right)$ amplitudes disappears at subsequent melting, as can be seen in Fig. 4. However, if a sample cooled after a long-term melting contacts the atmosphere, the above growth reappears. This must be the consequence of some contamination of Si surface at such a contact.

\section{References}

1. P.I. Baranskii, V.M. Babich, S.V. Svechnikov et al., Microgravity and superhigh vacuum - specific components of a technological environment and new capabilities of superconductor technology // Space science and technology 8(4), p. 96-99 (2002).

2. Yu.G. Ptushinskii, Low-temperature adsorption of gases on metal surface // Fizika nizkikh temperatur 30(1), p. 3-37 (2004) (in Russian).

3. V.A. Chuikov, V.D. Osovskii, Yu.G. Ptushinskii et al., Low-temperature studies of adsorption of gases on metal // Surf. Sci. 213(2), p. 359-370 (1989).

4. V.D. Osovskii, Yu.G. Ptushinskii, V.G. Sukretnyi et al., Adsorption of oxygen and hydrogen on the surface of a silicon single-crystal at low temperatures (up to $5 \mathrm{~K}$ ) // Fizika nizkikh temperatur 19(5), p. 570-578 (1993) (in Russian).

5. Vacuum engineering / Ed. E.S. Frolov and V.E. Minaichev. Mashinostrojenie, Moscow, 1985, p. 269-279 (in Russian).

6. J. Weston, Superhigh vacuum engineering. Mir, Moscow, 1988, p. 245 (in Russian). 